\title{
Quantitative Classification of [WC] Nuclei of Planetary Nebulae
}

\author{
A.Acker $^{1}$, M. Parthasarathy ${ }^{1,2}$, W.Leindecker ${ }^{1}$, J.Koeppen ${ }^{1}$ and B.Stenholm ${ }^{3}$ \\ ${ }^{1}$ Observatoire de Strasbourg, 11, rue de l'Universite, 67000 Strasbourg, France; \\ ${ }^{2}$ Indian Institute of Astrophysics, Bangalore - 560034, India; \\ ${ }^{3}$ Lund Observatory, Box 43, 22100 Lund, Sweden
}

We have carried out spectral classification of 65 [WC] stars, using spectra obtained at ESO in 1994 - 1995 with a spectral resolution of 1500 and high signal to noise ratio. We quantify the visual classification, by using the line ratios of CIII, CIV, OV, OVI in the $530-580$ $\mathrm{nm}$ range, correlated with the blue CIII - IV lines. We measured the total strength of the above emission lines and derived the ratios of the emission line strengths. Using the stellar emission line ratios we classified about 32 CSPN as [WC] and about 30 as WELS which show only relatively weak CIV 5806 and CIV 4650 and He II 4686 stellar emission lines. The presence of CIV 5806 and the absence of CIII 5696 the WELS appear to belong to the [WC3] type. However the OV lines are very weak or absent. Some stars are reclassified, and 4 stars are found in the [WC5-7] gap. We classified 34 CSPN as [WC]. The [WC2] CSPN NGC 2867 and PB 6 show narrow and weak He II 5412 absorption feature. Based on the relative strengths of the stellar emission features NGC 2867 [WC 2], PB 6 [WC2] and M1-61 [WC4] appear to be related to WELS ( Weak Emission Line Central Stars of Planetary Nebulae ). The observed spectral characteristics of the central stars of these PN suggest that they are related to [WC] - PG 1159 type of objects.

We find 30 [WC] CSPN to be WELS. The spectra of the central stars of these PN have relatively weak and narrow stellar emission features of C IV 5806, C IV - He II 4650 4686. The OV and CIII stellar emission features are absent. The presence of C IV $5806 \mathrm{~A}$ feature and the absence of CIII 5696 suggest the spectral type for all these objects to be in the range of [WC 3]. In several of these CSPN we find absorption lines of He II 5412. The CIV - He II 4650-4686 and CIV 5806 stellar emission features of these so called WELS are similar to that found in the PG 1159 objects. We suggest that the WELS found by us and the WELS listed by Tylenda et al ( 1993 ) are [WC] - PG 1159 type of objects. Recently, Schonberner and Napiwotzki ( 1990 ) showed that several CSPN are spectroscopically indistinguishable from the PG 1159 pre-white dwarfs. The PG 1159 objects have broad HeII and CIV absorption trhough near 4650 - $4686 \mathrm{~A}$, but the O VI emission is very weak or absent. The 4650 - 4686 A and 5806 A spectral feature of the WELS found by us are similar to that of Abell 30, Abell 78, NGC 6567, NGC 6578 and Longmore 4 at outburst, which are considered as [WC]-PG 1159 type ( Hamann 1996 ). We conclude that the WELS are most likely [WC]-PG 1159 objects. 